\title{
Content Based Image Retrieval using Feature Extraction Technique
}

\author{
Nandkumar S. Admile, Jagadish Hallur, Anup S.Vibhute, Akshay A.Jadhav, Vijay S.Bhong
}

\begin{abstract}
Now days the image processing can be used in various areas such as in Agriculture, in Health care system also for security purpose. In case of Crime investigation the image processing can be used to identify the particular suspect from an available dataset for that purpose an image retrieval technique is presented in this paper. For image retrieval number of techniques is available. In earlier days Block Truncation Coding is used but due its some disadvantage feature extraction method is used. Using DDBTC technique two features are derived. The first feature as Color Co-occurrence Features (CCF) obtained using color quantizes features such as Bit Pattern Feature (BPF) is derived from Bitmap image. The five different distance metrics are used to measure the similarity between two images. The simulated results shows proposed Technique can shows the better result in the form of Average Precision rate (APR) and Average Recall Rate (ARR) as compared to other techniques.
\end{abstract}

Keywords: Average Recall Rate Average Precision Rate, Bit pattern feature, color co-occurrence feature, Dot diffused block truncation coding.

\section{INTRODUCTION}

In recent years in the field of image processing number of methods and techniques are developed. In every field such as Bio-medical, engineering image processing is widely used. Now a days in the field of medical image processing can be used to detect the brain cancer and tumour.Also the image processing find the application in computer vision. In current days the database of images can be rapidly increases. Due to these we face the problem of detection and identification of particular image from large dataset. Hence to overcome such type of problem Image Retrieval is developed. The image retrieval technique can easily detect a particular image from a large dataset, but in initial stage it having number of problems to retrieve an image. Hence content based and BTC based Techniques are used along with image retrieval. In 1979 [1] Mitchell and Delp introduces first Block Truncation Coding

Revised Manuscript Received on December 30, 2019.

* Correspondence Author

Nandkumar Sushen Admile, Department of E\&TC Engg.College of Engineering, Pandharpur, admile.kumar@gmail.com

Jagdish S.Hallur, Department of E\&TC Engg.College of Engineering, Pandharpur, jshallur@coe.sveri.ac.in

Dr.Anup S.Vibhute, Department of E\&TC Engg.College of Engineering, Pandharpur, ,asvibhute@coe.sveri.ac.in

Akshay A.Jadhav, Department of E\&TC Engg.College of Engineering, Pandharpur, ,aajadhav@coe.sveri.ac.in

Vijay S.Bhong, Department of E\&TC Engg. College of Engineering, Pandharpur, vsbhong@coe.sveri.ac.in

(c) The Authors. Published by Blue Eyes Intelligence Engineering and Sciences Publication (BEIESP). This is an open access article under the CC BY-NC-ND license (http://creativecommons.org/licenses/by-nc-nd/4.0/)
(BTC). In 1987the author Raina and Udpikar [2] uses BTC-VQ.Using this technique 8:1 compression ratio is achieved. Author Yiyan [3] introduces BTC-VQ-DCT technique. The DCT coding is implemented to remove both the inter and intra block redundancy. The compression ratio is about 10:1In 2003 author G.Qui [4] uses the Block Truncation coding technique and derives the two features namely BCCM and BPH.Author N. Jhanwara [5] achieves Better result than CCM. In 2012 author M. Subrahmanyam [6] derives a new algorithm for retrieval of images which shows some enhancement in performance as compared to LBP. In 2013 author uses [7] structure elements' descriptor (SED) texture descriptor to represent and describe local features of images. The method proves the performance of the SED is better than various texture based descriptors. In 2013 author [8] designs image indexing technique using JPEG images. Vector quantization techniques (VQ) is used to extract the image features and K-means clustering algorithm is used to generate the codebook. In [9] author describes different image retrieval techniques. In [10] author extract three features whereas in [11] author extract two features using DDBTC technique It achieves the better retrieval performance as compare existing techniques methods

\section{PROPOSED METHOD}

\section{A. System Design Flow:}

The Structured diagram of system design flow is mentioned in fig. 1

\section{B. Dot Diffusion Block Truncation Coding:}

A sample image is used to derive the minimum and maximum quantizer. In this assume at position (i, j) an $\mathrm{f}(\mathrm{i}$, j) be the image block Where $\mathrm{i}=1,2 \ldots \ldots \ldots . \mathrm{M} / \mathrm{m}$ and $\mathrm{j}=$ $1,2 \ldots \ldots . . \mathrm{N} / \mathrm{n}$. The color images are in RGB color space.

$$
\text { Qmin }=\left\{\min \mathrm{f}_{\mathrm{R}}(\mathrm{x}, \mathrm{y}), \min \mathrm{f}_{\mathrm{G}}(\mathrm{x}, \mathrm{y}), \min \mathrm{f}_{\mathrm{B}}(\mathrm{x}, \mathrm{y})\right\}
$$

$\mathrm{Qmax}=\{\max \mathrm{fR}(\mathrm{x}, \mathrm{y}), \max \mathrm{fG}(\mathrm{x}, \mathrm{y}), \max \mathrm{fB}(\mathrm{x}, \mathrm{y})\}$

In this Red, Green and blue color are represented by R, $\mathrm{G}$ and $\mathrm{B}$.The grey image is obtained from color image using $\mathrm{f}^{\wedge}(\mathrm{x}, \mathrm{y})=1 / 3\left[\mathrm{f}_{\mathrm{R}}(\mathrm{x}, \mathrm{y})+\mathrm{f}_{\mathrm{G}}(\mathrm{x}, \mathrm{y})+\mathrm{f}_{\mathrm{B}}(\mathrm{x}, \mathrm{y})\right]$

The Bitmap image is also computed using:

$$
b_{m}=1 \text {; if, } f^{\wedge}(x, y) \geq f(i, j)
$$




\section{Content Based Image Retrieval using Feature Extraction Technique}

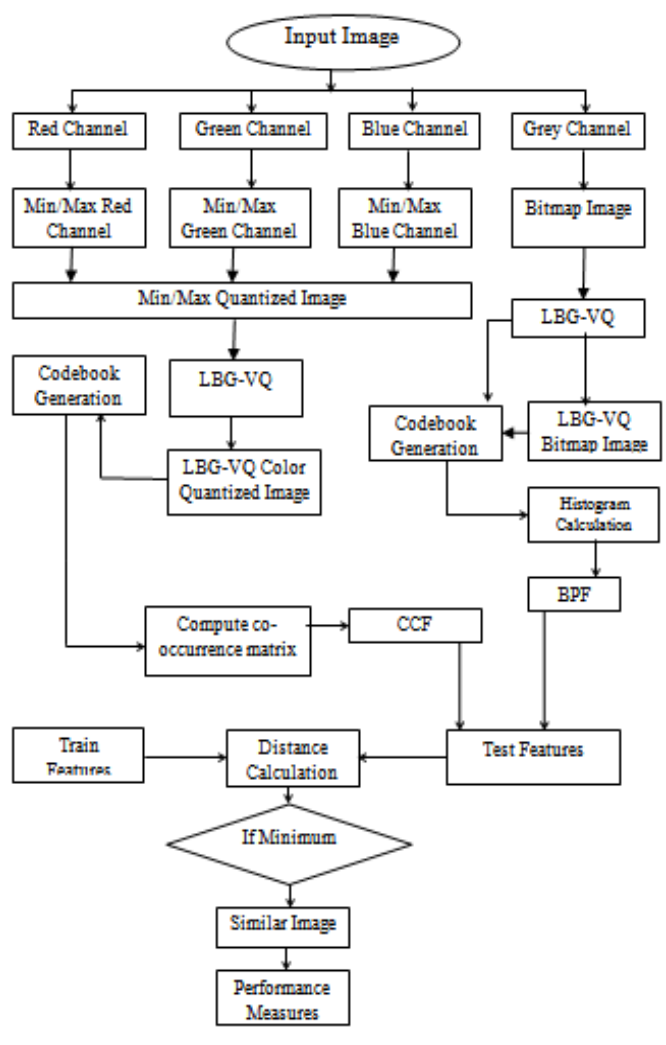

Fig.1.Flow Graph

III. FEATURE EXTRACTION

\section{A. Color Co Occurrence Feature (CCF):}

To obtain the color co-occurrence feature the co-occurrence matrix is generated with the help of codebook and LBG-VQ color quantized image as shown in Fig 3.

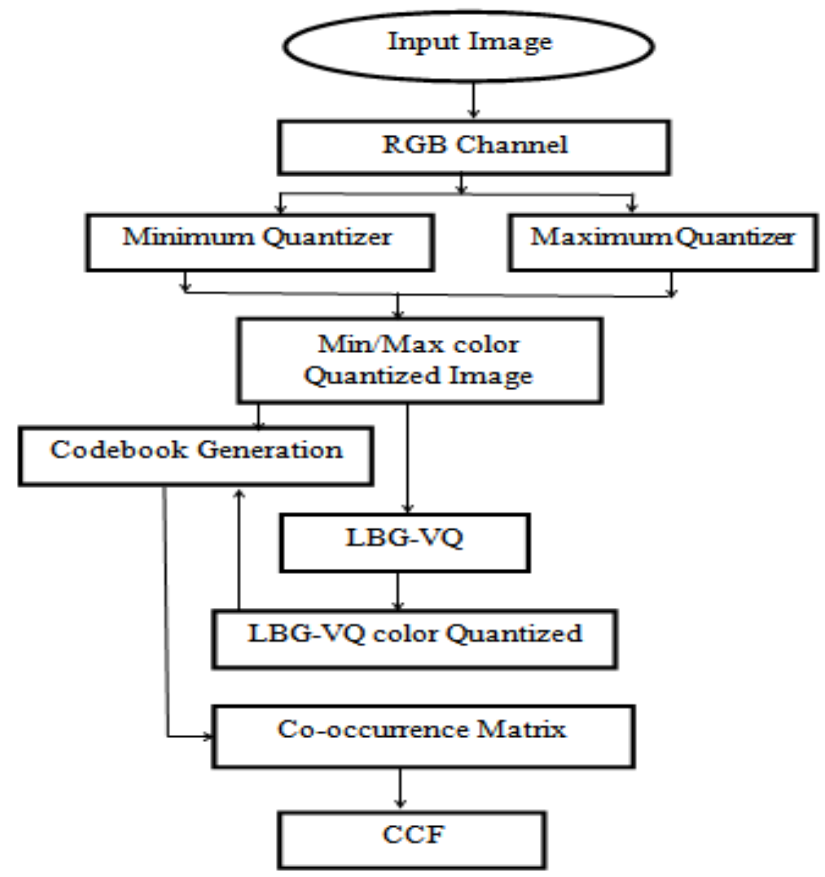

Fig.2. Color Co Occurrence Feature

\section{B. Bitmap Pattern Feature (BPF):}

To compute Bit Pattern Feature gray scale image is used.
Using grey image bitmap image is generated. From codebook and LBG-VQ bitmap image Histogram is calculated. The Design flow is shown in Fig 4.

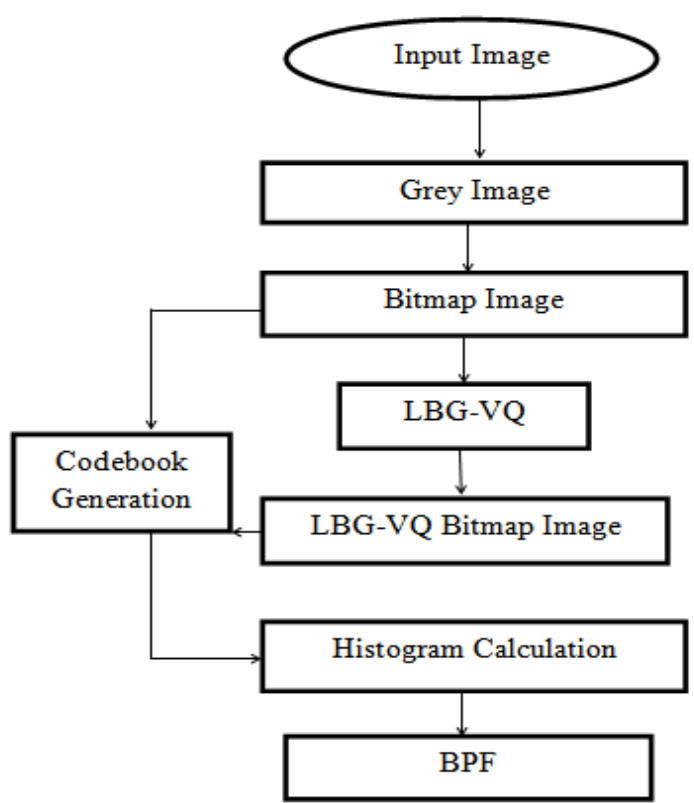

Fig.3. Bitmap Pattern Feature

\section{DISTANCE METRICS}

The various distances are given by: $1 . \mathrm{L}_{1}$ distance $\delta($ query, target $)=a_{1} \sum_{k=1}^{N \min }\left|C H F_{\min }^{\text {query }}(k)-C H F_{\min }^{\text {target }}(k)\right|$

$$
\begin{aligned}
& +a_{2} \sum_{k=1}^{N \max }\left|C H F_{\min }^{q u e r y}(k) C H F_{\min }^{\text {target }}(k)\right| \\
& +a_{3} \sum_{k=1}^{N b}\left|B P F^{\text {query }}(k)-B P F^{\text {target }}(k)\right|
\end{aligned}
$$

2.L distance

$$
\begin{aligned}
\delta(\text { query, target }) & =\left[a_{1} \sum_{k=1}^{N \min }\left(C H F_{\text {min }}^{\text {query }}(k)-C H F_{\text {min }}^{\text {target }}(k)\right)^{2}\right. \\
& +a_{2} \sum_{k=1}^{N \max }\left(C H F_{\text {min }}^{\text {query }}(k)-C H F_{\text {min }}^{\text {target }}(k)\right)^{2} \\
& \left.+a_{3} \sum_{k=1}^{N b}\left(B P F^{\text {query }}(k)-B P F^{\text {target }}(k)\right)^{2}\right]^{1 / 2}
\end{aligned}
$$

3. $\mathrm{x}^{2}$ Distance

$$
\begin{aligned}
& \delta(\text { query, target })= \\
& \quad \alpha_{1} \sum_{k=1}^{N_{\min }}\left(\frac{C H F_{\min }^{\text {query }}(k)-C H F_{\min }^{\text {target }}(k)}{C H F_{\min }^{\text {query }}(k)+C H F_{\min }^{\text {target }}(k)+\varepsilon}\right)^{2} \\
& +\alpha_{2} \sum_{k=1}^{N_{\max }}\left(\frac{C H F_{\max }^{\text {query }}(k)-C H F_{\max }^{\text {target }}(k)}{C H F_{\max }^{\text {query }}(k)+C H F_{\max }^{\text {target }}(k)+\varepsilon}\right)^{2} \\
& +\alpha_{3} \sum_{k=1}^{N_{b}}\left(\frac{B P F^{\text {query }}(k)-B P F^{\text {target }}(k)}{B P F^{\text {query }}(k)+B P F^{\text {target }}(k)+\varepsilon}\right)^{2}
\end{aligned}
$$


4. Fu Distance:

$\delta($ query, target $)=\frac{L_{2} \text { distance }}{\left|\mathrm{CHF}_{\min }+\right| \mathrm{CHF} F_{\text {max }}+|\mathrm{BPF}|}$

5. Modified Canberra distance.

$$
\begin{aligned}
& \delta(\text { query, target })=a_{1} \sum_{k=1}^{N \min } \frac{\left|C H F_{\min }^{\text {query }}(k)-C H F_{\min }^{\text {target }}(k)\right|}{\operatorname{CHF}_{\min }^{\text {query }}(k)+C H F_{\min }^{\text {target }}(k)+\varepsilon} \\
& +a_{2} \sum_{k=1}^{N \max } \frac{\left|C H F_{\max }^{\text {query }}(k)-C H F_{\max }^{\text {target }}(k)\right|}{\operatorname{CHF}_{\max }^{\text {query }}(k)+C H F_{\max }^{\text {target }}(k)+\varepsilon} \\
& +a_{3} \sum_{k=1}^{N b} \frac{\left|B P F^{\text {query }}(k)-B P F^{\text {target }}(k)\right|}{B P F^{\text {query }}(k)+B P F^{\text {target }}(k)+\varepsilon}
\end{aligned}
$$

\section{SYSTEM PERFORMANCE}

The System performance determined using:

$$
\begin{aligned}
& A P R=\frac{1}{\mathbb{N t} L} \sum_{q=1}^{N t} \mathrm{Nq}(\mathrm{L}) \\
& A R R=\frac{1}{\operatorname{Nt~Nr}} \sum_{\mathrm{q}=1}^{\mathrm{Nt}} \mathrm{Nq}(\mathrm{Nr})
\end{aligned}
$$

\section{EXPERIMENTAL RESULTS}

A. Experimental Setup:

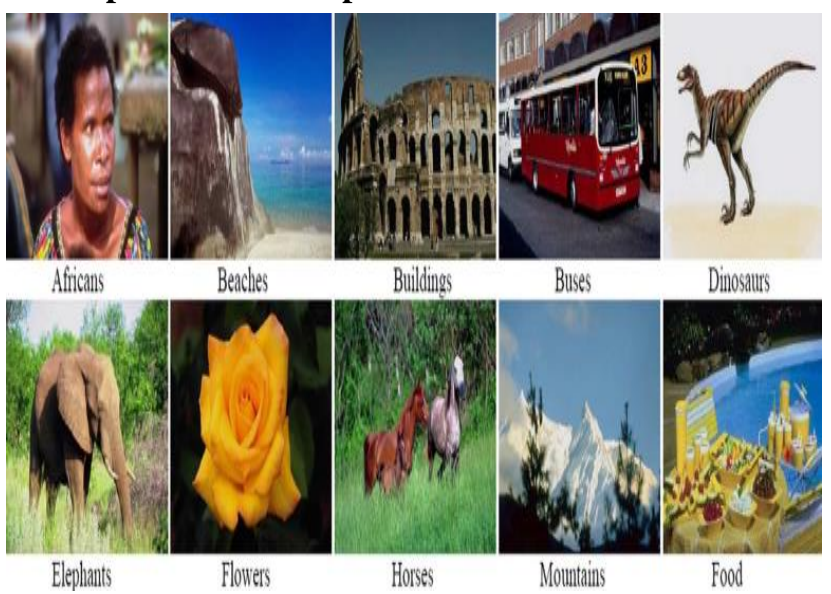

Fig.4. Corel Image Dataset

To calculate the results Corel 1000 image Dataset is used. Fig.4. shows standard dataset. To perform the image retrieval task 20 images of each class are selected

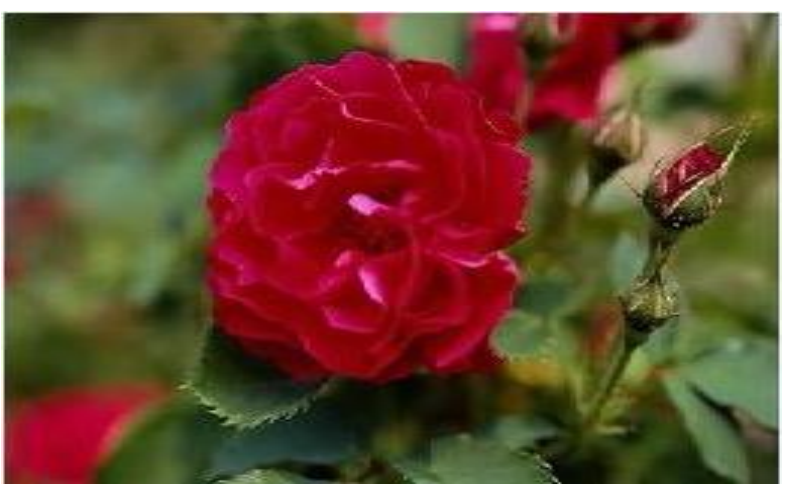

Fig.5. Input color image

A sample image from Corel dataset shown in Fig.5. Using LBG vector quantization two images are obtained as shown in fig.6.andin fig.7.

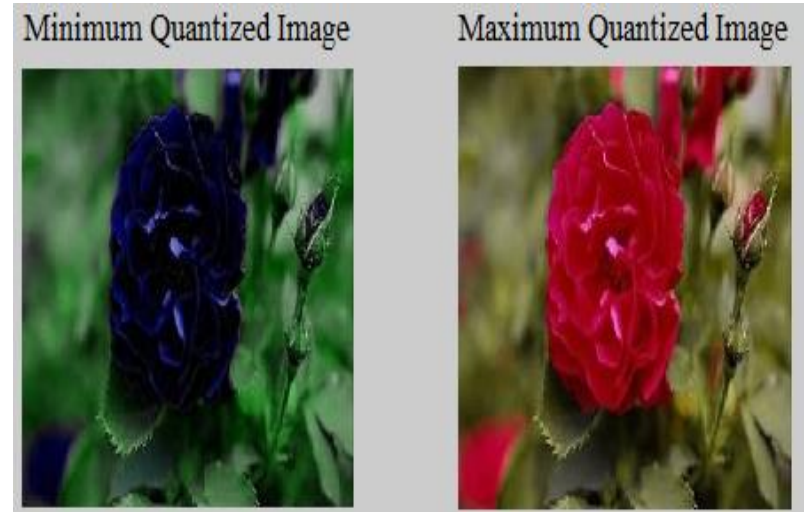

Fig. 6. Min. and Max. Quantized Image

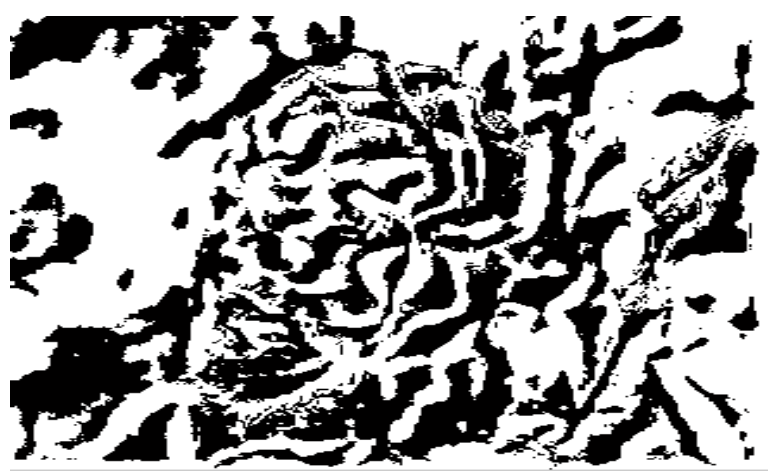

Fig.7. Bitmap Image

The fig. 8 and fig. 9 are obtained using color co-occurrence matrix.

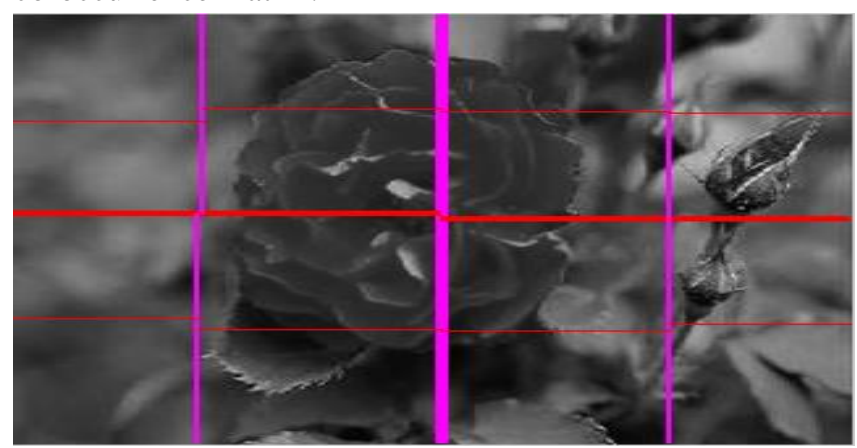

Fig.8. Ordered Matrix with centered Points

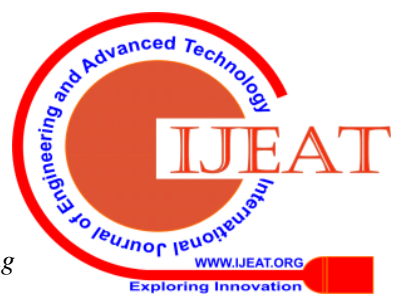


Content Based Image Retrieval using Feature Extraction Technique

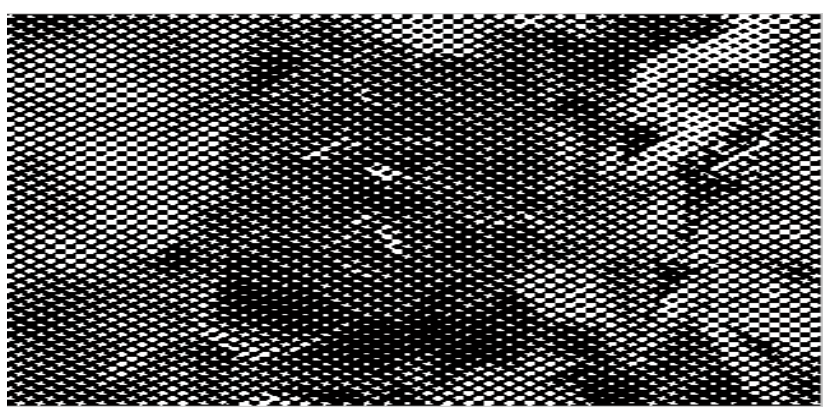

Fig.9.Diagonal Ordered Matrix with Balanced

Centered Point

Similarity of images is measured by using different distance metrics. Retrieved images using modified Canberra distance shown in fig. 10

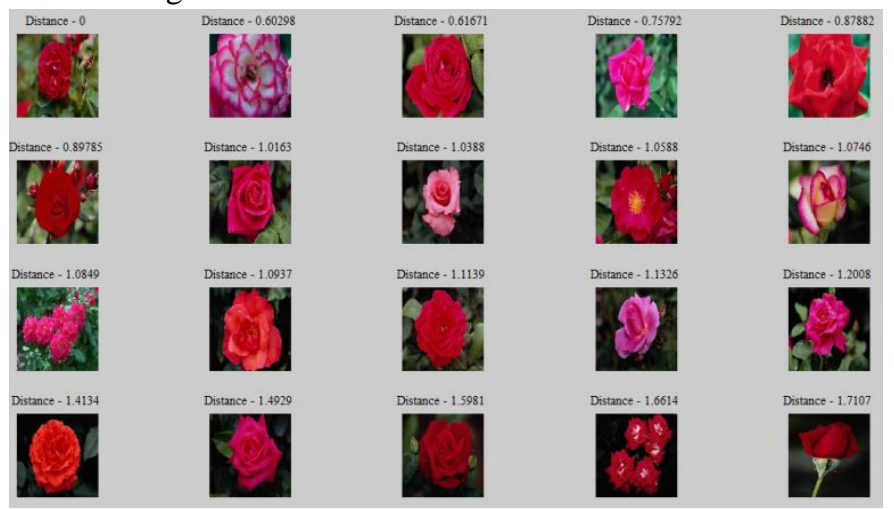

Fig.10. Retrieved Images

The image retrieval task can be performed by using five different distance metrics. Out of which modified Canberra distance shows the better accuracy listed in Table. I, II, III.

Table. I.

\begin{tabular}{|c|c|c|c|c|}
\hline Class & L1 & L1 & L2 & L2 \\
\hline & APR & ARR & APR & ARR \\
\hline African & 0.6875 & 60.19 & 0.6875 & 60.19 \\
\hline Beach & 0.6875 & 65.19 & 0.6875 & 65.19 \\
\hline Building & 0.6875 & 70.19 & 0.6875 & 70.19 \\
\hline Bus & 0.8125 & 80.19 & 0.8125 & 80.19 \\
\hline Dinosaur & $0 . .8750$ & 90.19 & 0.8750 & 90.19 \\
\hline Elephant & 0.5625 & 60.19 & 0.5625 & 60.19 \\
\hline Flower & 0.8125 & 70.19 & 0.8125 & 70.19 \\
\hline Food & 0.8750 & 65.19 & 0.8750 & 65.19 \\
\hline House & 0.6875 & 65.19 & 0.6875 & 60.19 \\
\hline Mountain & 0.9575 & 95.19 & 0.9375 & 95.19 \\
\hline
\end{tabular}

Table II

\begin{tabular}{|c|c|c|c|c|}
\hline Class & X2 & X2 & Fu & Fu \\
\hline & APR & ARR & APR & ARR \\
\hline African & 0.8750 & 90.19 & 0.6875 & 60.19 \\
\hline Beach & 0.8750 & 90.19 & 0.6875 & 65.19 \\
\hline Building & 0.8750 & 90.19 & 0.6875 & 70.19 \\
\hline Bus & 0.9375 & 95.19 & 0.8125 & 80.19 \\
\hline Dinosaur & 0.9375 & 95.19 & 0.8750 & 90.19 \\
\hline Elephant & 0.9375 & 90.19 & 0.5625 & 60.19 \\
\hline Flower & 0.8750 & 90.19 & 0.8125 & 70.19 \\
\hline Food & 0.9375 & 90.19 & 0.8750 & 65.19 \\
\hline House & 0.8750 & 90.19 & 0.6875 & 60.19 \\
\hline Mountain & 0.9375 & 95.19 & 0.9375 & 75.19 \\
\hline
\end{tabular}

Table III

\begin{tabular}{|c|c|c|}
\hline & \multicolumn{2}{|c|}{ Modified Canberra Distance } \\
\hline & APR & ARR \\
\hline African & 0.8750 & 90.19 \\
\hline Beach & 0.8750 & 90.19 \\
\hline Building & 0.8750 & 90.19 \\
\hline Bus & 0.9375 & 95.19 \\
\hline Dinosaur & 0.9375 & 95.19 \\
\hline Elephant & 0.9375 & 90.19 \\
\hline Flower & 0.9375 & 95.19 \\
\hline Food & 0.9375 & 95.19 \\
\hline House & 0.8750 & 90.19 \\
\hline Mountain & 0.9375 & 95.19 \\
\hline
\end{tabular}

\section{GRAPHICAL REPRESENTATION}

Performance Image Retrieval is determined in form of APR and ARR listed in fig.11. And fig.12. 


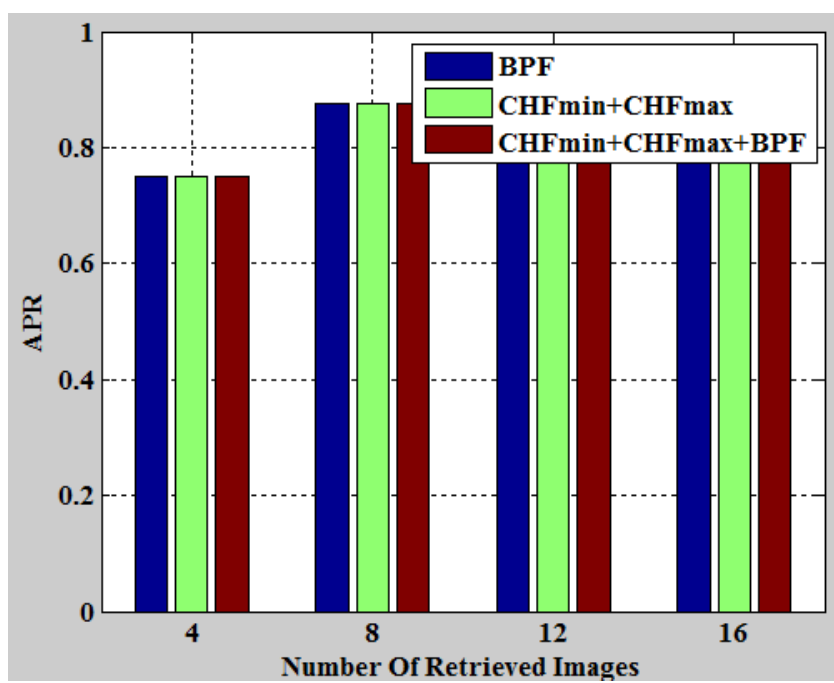

Fig.11. Performance Graph of APR

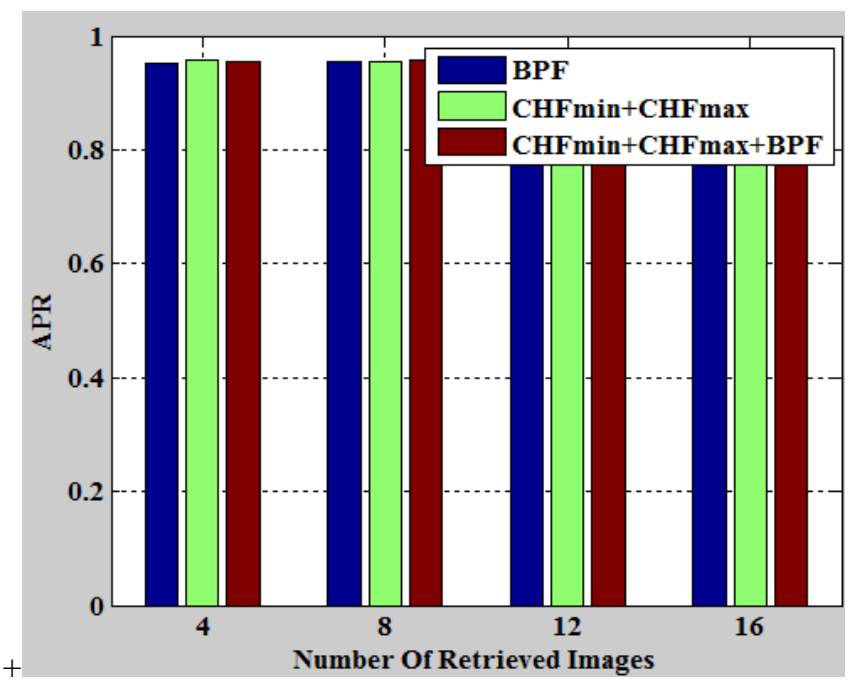

Fig.12.Performance Graph of ARR

The accuracy of Image Retrieval is drawn in graph 3

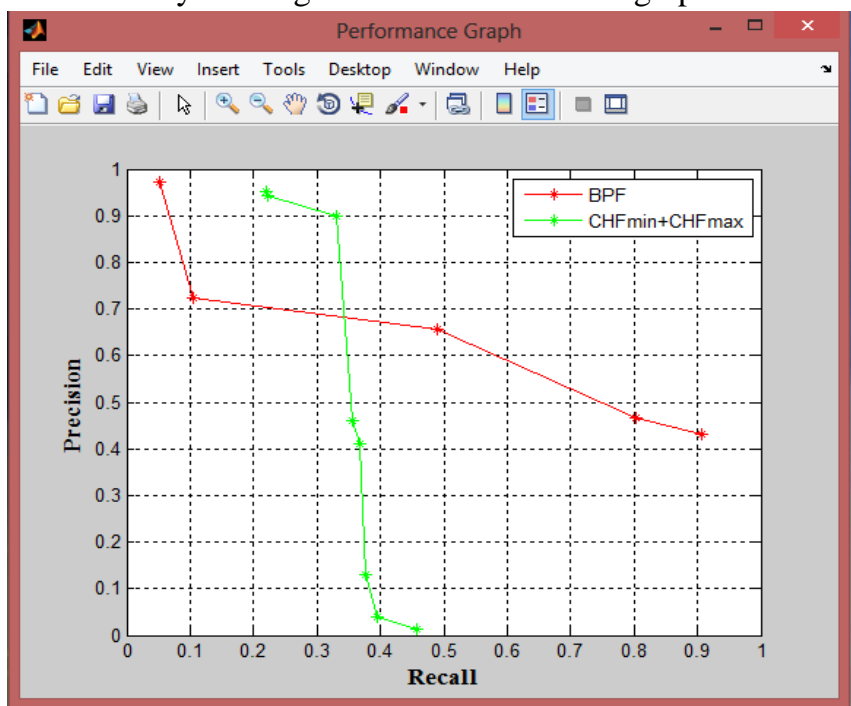

Fig.13. Precision vs. Recall Performance

\section{CONCLUSION}

To perform the Experiment DDBTC method and two image features are used. BPF recognize the contents of image and CCF represents color distribution and image brightness. The simulated results shows better accuracy. In the future work, instead of Corel dataset another dataset can also employed. The video retrieval can also use to perform image retrieval task. For this sequence of images can be considered as a video.

\section{REFERENCES}

1. E. J. Delp and O. R. Mitchell, "Image coding using block truncation Coding," IEEE Trans. Comm., vol. COM-27, no. 9, pp. 1335-1342, Sep. 1979.

2. V. Udpikar and J. Raina, \BTC image coding using vector quantization," IEEE Trans.Commun., vol. 35, no. 3, pp. 352 \{356, Mar. 1987.

3. Y. Wu and D. C. Coll, \BTC-VQ-DCT hybrid coding of digital images," IEEE Trans. Commun., vol. 39, no. 9, pp. 1283\{1287, Sep. 1991.

4. G. Qiu, "Color image indexing using BTC," IEEE Trans. Image Process. vol. 12, no. 1, pp. 93-101, Jan. 2003.

5. N. Jhanwar, S. Chaudhurib, G. Seetharamanc, and B. Zavidovique,IContent based image retrieval using motif co-occurrence matrix," Image Vis. Comput., vol. 22, pp. 1211\{1220, Dec. 2004.

6. M. Subrahmanyam, R. P. Maheswari, and R. Balasubramanian, \Expert system design using wavelet and color vocabulary trees for image retrieval," Expert Syst. Appl., vol.39, no. 5, pp. 5104\{5114, 2012.

7. W. Xing Yuan and W. Zongyu, \A novel method for image retrieval based on structure element's descriptor, "vol. 24, no. 1, pp. 63\{74, 2013.

8. P. Pour Sistani, H. Nezamabadi-pour, R. A. Moghadam, and M. Saeed, Image indexing and retrieval in JPEG compressed domain based on vector quantization," Math. Comput. Modeling, vol. 57, no. 5\{6, pp.1005 \{1017, 2013.

9. Nandkumar Sushen Admile, A survey on Different Image Retrieval Techniques, IRJET International Research Journal of Engineering and Technology, Volume 5, Issue 10, October 2018

10. Nandkumar S. Admile and Prof. Rekha Dhawan, Content Based Image Retrieval Using Feature Extracted from Dot Diffusion Block Truncation coding, IEEE International conference on communication and Electronics Systems (ICCES-2016) Coimbatore, India, 21-22 October 2016. DOI: 10.1109/CESYS.2016.7889864, INSPEC Accession Number: 16776462

11. Nandkumar S. Admile Image Retrieval Based on Block Truncation coding, IEEE International conference on communication and Electronics Systems (ICCES-2018)Coimbatore, India, 15-16 October 2018.

DOI: 10.1109/CESYS.2018.8724097, INSPEC Accession

Number: 18723995

\section{AUTHORS PROFILE}

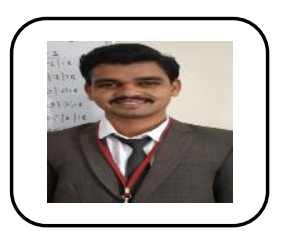

Nandkumar Sushen Admile, working as assistant professor in Electronics and Tele-Communication Engineering Department from College of Engineering, Pandharpur,Maharashtra,India

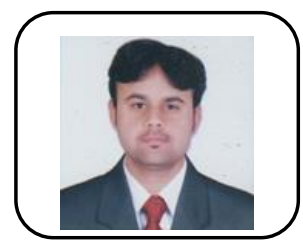

Jagadish S Hallur, working as assistant professor in Electronics and Tele-Communication Engineering Department from College of Engineering, Pandharpur, Maharashtra, India

Dr.Anup S.Vibhute, working as a Professor and Head in Electronics and Tele-Communication Engineering Department from College of Engineering, Pandharpur, Maharashtra, India

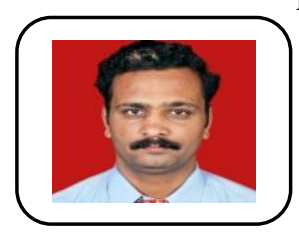

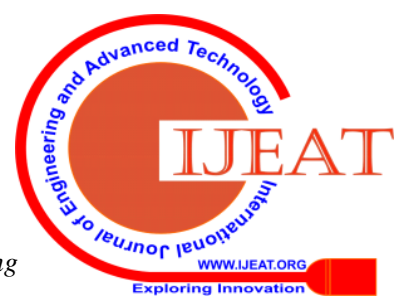




\section{Content Based Image Retrieval using Feature Extraction Technique}

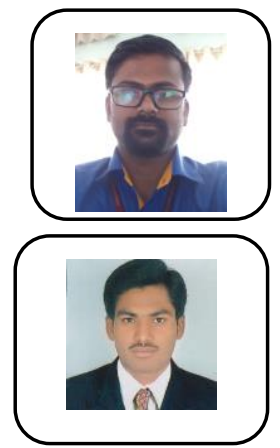

Akshay A.Jadhav, working as assistant professor in Electronics and Tele-Communication Engineering

Department from College of Engineering, Pandharpur, Maharashtra, India

Vijay S.Bhong, working as assistant professor in Electronics and Tele-Communication Engineering Department from College of Engineering, Pandharpur, Maharashtra, India 